\title{
Current Technology Development Efforts on the International X-Ray Observatory
}

\author{
David Robinson \\ NASA Goddard Space Flight Center \\ Greenbelt, MD 20771 \\ 301-286-9926 \\ david.w.robinson@nasa.gov
}

\begin{abstract}
The International X-ray Observatory (IXO) is a collaboration between NASA, ESA, and JAXA which is under study for launch in 2021. IXO will be a large 6600 kilogram Great Observatory-class mission which will build upon the legacies of the Chandra and XMM-Newton X-ray observatories. There is an extensive ongoing effort to raise the technology readiness level of the X-ray mirror from TRL 3 to TRL 6 in the next decade. Improvements have recently been made in the area of positioning and bonding mirrors on the nanometer scale and developing metals and composites with a matching coefficient of thermal expansion to the glass X-ray mirrors. On the mission systems side, the NASA reference design has been through a preliminary coupled loads analysis and a STOP analysis of the flight mirror assembly has been initiated. An impact study was performed comparing launching IXO on an Ariane 5 or a U.S. EELV. This paper will provide a snapshot of NASA's current observatory configuration and summarize the progress of these various technology and design efforts.
\end{abstract}

\section{TABLE OF CONTENTS}

1. INTRODUCTION . .1

2. CURRENT STATUS OF IXO .................................................3

3. RECENT IMPROVEMENTS IN MIRROR ALIGNING AND BONDING . .3

3. STRUCTURAL, THERMAL, OPTICAL ANALYSIS................4

4. OVERCOMING THERMAL EXPANSION PROBLEMS WITH NEW MATERIALS

5. PRELIMINARY COUPLED LoAdS ANALYSIS .....................9

6. LAUNCHER COMPARISON REFERENCES

. .9

\section{INTRODUCTION}

The International X-ray Observatory (IXO) is a collaboration between NASA, ESA, and JAXA which in the formulation phase. ${ }^{1} 2$ IXO combines elements from NASA's prior Constellation-X program [1] and ESA's XEUS program. IXO will be a large Great-Observatory class mission which will build upon the legacies of the Chandra and XMM-Newton X-ray observatories. Two major improvements in IXO over Chandra and XMM Newton are the high effective area for $\mathrm{X}$-ray photon collecting in the $1 \mathrm{keV}$ to $6 \mathrm{keV}$ range, and a high spectral

\footnotetext{
${ }^{1}$ U.S. Government work not protected by U.S. copyright.

2 IEEEAC paper \#1094, Version 3 updated 2011:01:11.
}

resolving power achieved with a micro-calorimeter X-ray detector cooled to 50 milliKelvin. The exciting science enabled by IXO includes exploration of black holes, growth and evolution of the largest structures in the universe and cosmic feedback. Details can be found in many other papers $[2,3]$ and will not be discussed here.

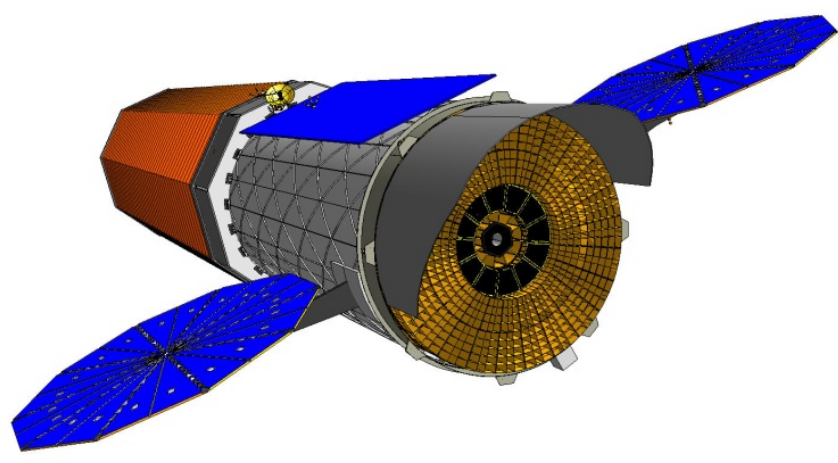

Figure 1 - View of IXO’s Optics Module side

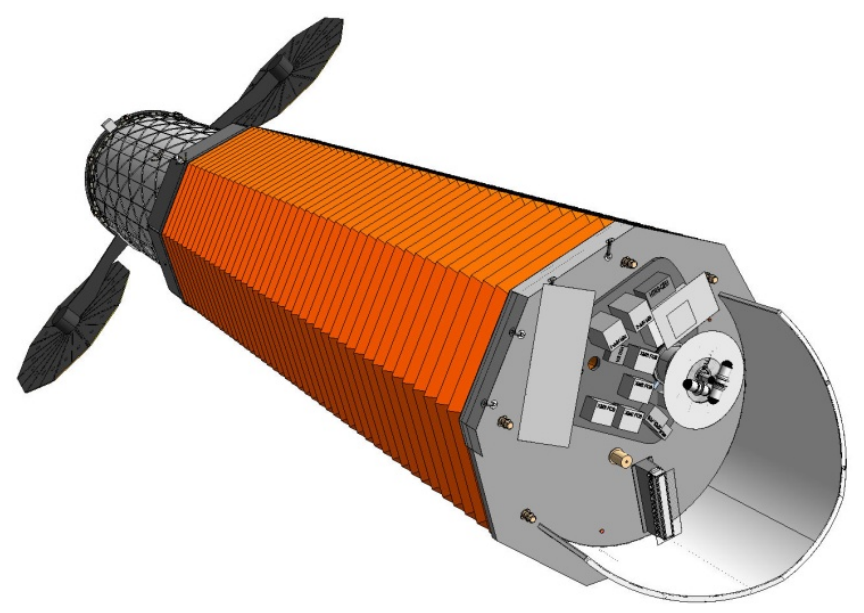

Figure 2 - View of IXO's Focal Plane Instrument side

IXO will have a launch mass in the neighborhood of 6600 $\mathrm{kg}$ and will be approximately 10 meters long and 4 meters in diameter in its launch configuration. It will fly on an Atlas 5 or an Ariane V rocket into an L2 halo orbit. To collect as many photons as possible, IXO will have a $3.1 \mathrm{~m}$ diameter Flight Mirror Assembly (FMA). Instead of the single large normal incidence primary mirror one finds in optical telescopes, the FMA will consist of nested concentric rings of mirrors which are nearly edge-on to the incoming X-ray 
photons. X-ray photons glance off the mirror surface at a very shallow angle and are focused 20 meters downstream at the instrument module end of the spacecraft. In the baseline concept, the FMA has approximately 350 concentric shells of glass, and 13,400 individual pieces of curved glass segments each $0.40 \mathrm{~mm}$ thick. Another X-ray focusing concept under development by ESA consists of "micropore" optics [4]. Both concepts present major technological challenges to the IXO mechanical team, particularly in maintaining alignment and ensuring survivability during launch.

A major science driver for the observatory is a long focal length since that enables more photon collecting capability at the higher energy ranges. A focal length of 20 meters was selected for IXO as a reasonable balance between science needs and engineering constraints. No rocket fairing is large enough to fit a 20 meter long observatory, so IXO will have a deployable metering structure between the spacecraft bus and the instrument module.

The FMA is the huge $\mathrm{x}$-ray collecting and focusing device for the X-ray telescope. It weighs about $1700 \mathrm{~kg}$ and is arguably the largest design driver of the observatory. The FMA's Wolter-I X-ray telescope optical design consists about $14,000 \quad 0.4 \mathrm{~mm}$ thick glass mirror segments densely packed into a $3.1 \mathrm{~m}$ diameter primary structure and supported with micron level accuracy and stability.

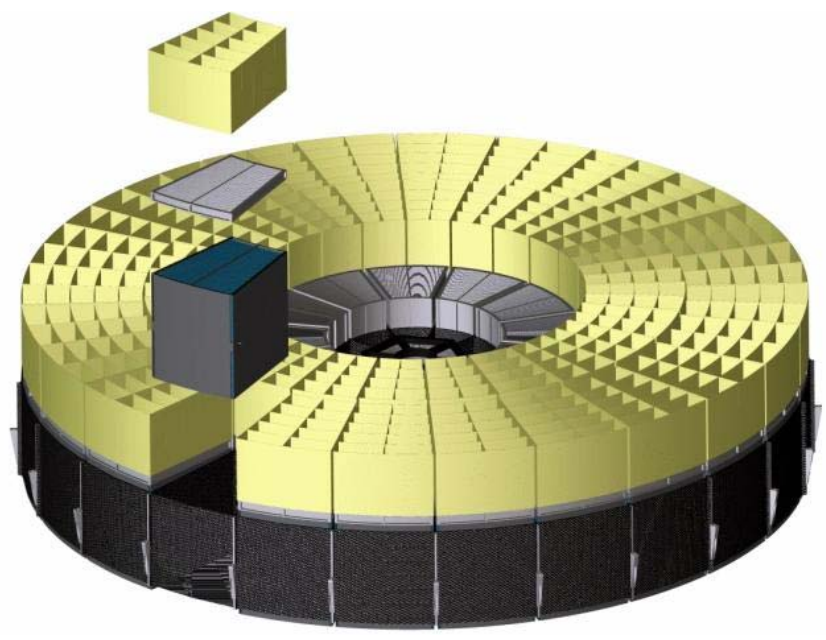

Figure 3 - Flight Module Assembly and spacecraft adapter.

A research and development program at NASA Goddard Space Flight Center has been in place for several years to determine the best way to form the glass mirrors and align them into a flight-like structure. The current concept involves placing flat glass sheets over a highly polished and figured mandrel and heating them to $\sim 600 \mathrm{C}$ at which temperature the glass slumps onto the mandrel and takes its shape. After cooling, the glass is removed, held in some fashion to reduce the effects of gravity, and bonded at the edges to a mirror module structure.
The primary structure of the FMA looks like a wagon wheel. It is currently envisioned to have 24 spokes which intersect at a central hub. Around the spokes are five rings. Modules will be kinematically mounted to the wagon wheel structure in the three outer rings. The inner ring will hold a high energy x-ray mirror assembly.

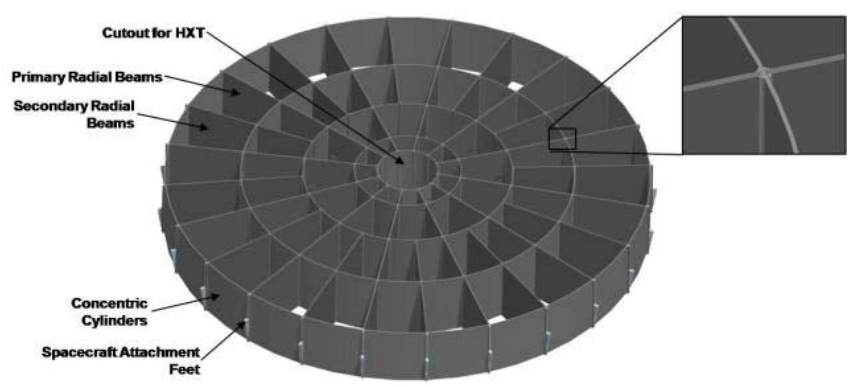

Figure 4 - Current FMA primary Structure concept

The mirror module is the basic building block of the FMA. A module is a wedge shaped housing which contains about 240 glass segments and weighs about $20 \mathrm{~kg}$. The current FMA design contains 24 modules around the FMA perimeter to form the outer ring. There are another 24 modules in the middle ring and 12 in the inner ring.

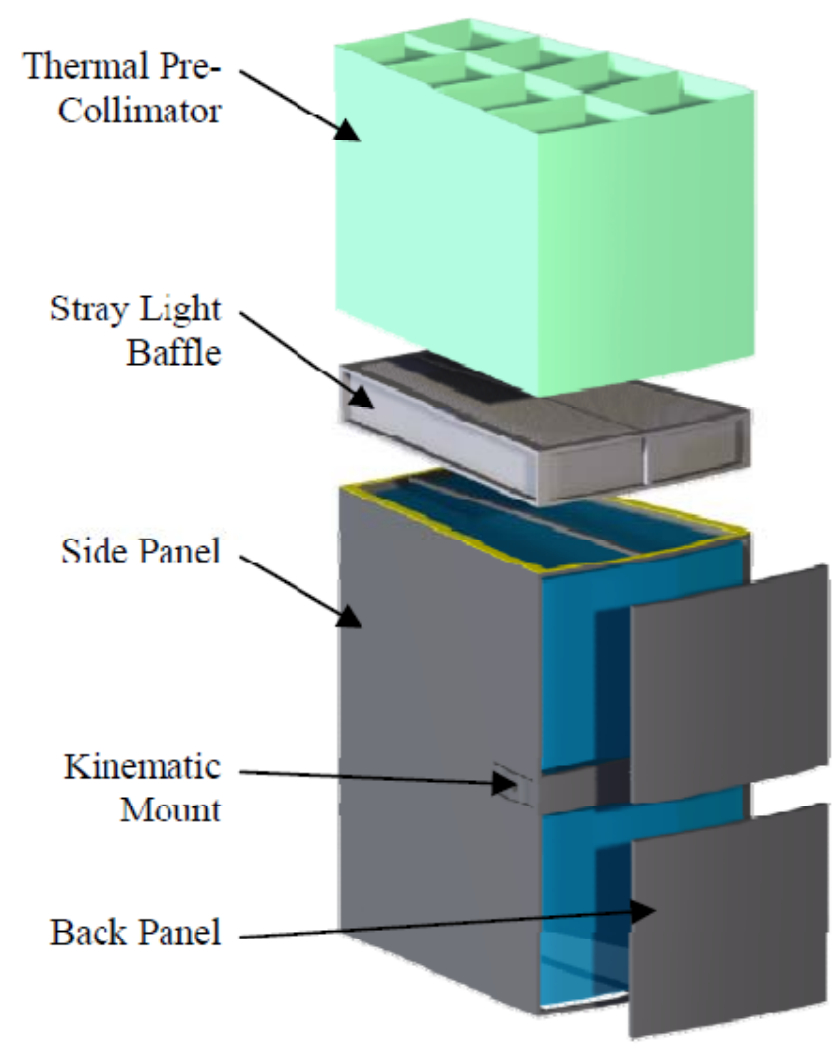

Figure 5 - Exploded View of a Mirror Module 
The FMA will be fastened to the spacecraft adapter ring in at least 6 locations. The spacecraft adapter ring is essentially a 3.2 meter diameter aluminum cylinder which attaches to the payload adapter on one side and the FMA and metering structure on the other side and it is the major structural component of the "Optics Module." The Optics Module consists of the FMA, spacecraft adapter, thermal control system, deployable covers, and a telescope aspect determination system.

\section{CURrent Status OF IXO}

In August 2010 IXO was ranked $4^{\text {th }}$ by the National Academy of Science's Astrophysics Decadal Survey [8] of all astrophysics space projects. This ranking was the result of an extensive pre-Phase A work for several years leading up to the submission of the proposal and subsequent followon requests for information. An independent cost estimation of approximately $\$ 5$ billion was performed by the Aerospace Corporation. This high cost was one of the major reasons IXO was not selected ahead of others including the dark energy mission WFIRST and the gravity wave mission LISA. While the ranking of $4^{\text {th }}$ is not as high as the project would have wished, it does keep IXO alive as a future major project. The Decadal report advised NASA to continue funding technology development on the mirror assembly and instruments during the next decade and take other steps in order to reduce the cost of the mission.

ESA is in the middle of a similar mission ranking exercise and has submitted an IXO proposal to the Cosmic Visions committee for L-class missions. Final selections are due in the latter part of 2011. If ESA decides to place IXO ahead of others in the L-class category then NASA may revisit the funding priority of IXO.

In the meantime, IXO continues to work on technology development particularly of the Flight Mirror Assembly. The FMA Technology Readiness Level (TRL) at the time of proposal submission was TRL 3. Currently it is at TRL 4 due to extensive opto-mechanical development efforts underway at Goddard.

\section{RECENT IMPROVEMENTS IN MIRROR ALIGNING AND BONDING}

FMA technology efforts have been chronicled in many publications including references 2, 3, and 6 in the references and these will not be duplicated here. The latest jump from TRL 3 to TRL 4 occurred in the summer and fall of 2010 with the successful bonding of an X-ray mirror pair into a housing and testing that pair in an X-ray facility with an optical error of less than 15 arc-seconds Half-Power Diameter (HPD).

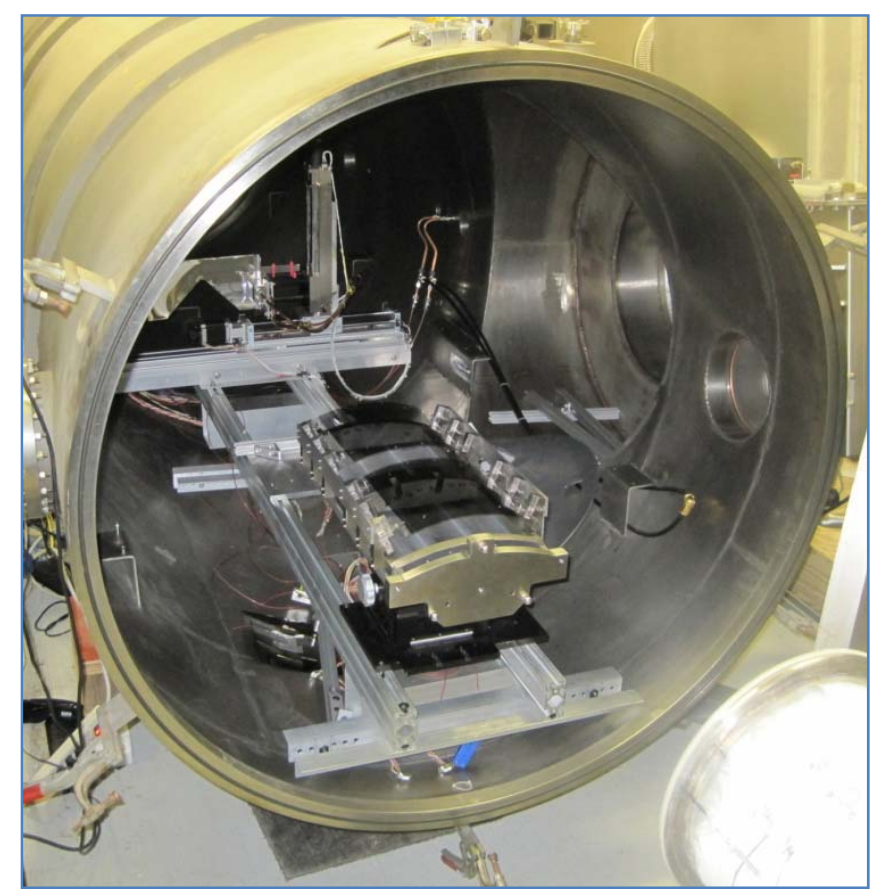

Figure 6 -X-ray Chamber with X-ray Mirror Pair installed

There are so many issues when dealing with $0.4 \mathrm{~mm}$ thick Xray mirrors that years ago it was difficult to know where to begin. The central problem is that the thin glass is very flexible and begins to lose its precise optical curvature when forces on the order of 1 milli-newton are applied. Simple operations such as placing and bonding a mirror into a housing become difficult since the very act of bonding a mirror induce forces into the mirror that distort it. Current $\mathrm{X}$-ray mirrors such as on the Astro-E2, XMM-Newton, and Nustar missions use thin metallic or glass X-ray mirror foils and achieve no better than about 40-50 arc-seconds HPD error [5]. The Chandra X-ray telescope uses several inchthick ground glass mirror shells with outstanding resolution on the order of 0.5 arc-seconds HPD, but with corresponding heavy mass penalty. IXO seeks to bridge the gap between heavy high resolution mirrors and lightweight but poor resolution mirrors.

The process of slumping a mirror segment to its optical prescription is an important initial step in creating the FMA. Currently the slumping lab at Goddard is producing mirrors in the neighborhood of 4-5 arc-seconds HPD error and progress is ongoing. The key issues are how to economically polish a mandrel to a figure error in the 1 arcsecond or less range and how to ensure that the mirror conforms to this shape but not sticks to the mandrel after cooling. Details of the challenges of this process can be found in reference 7 . Once the mirrors are obtained from the slumping lab they are installed into a housing.

In order to achieve TRL 4, a mirror housing simulator was built to fit into an optical alignment station. The mirror housing simulator is a simplified version of a mirror module that can hold only a few primary and secondary mirror pairs. 
The optical station consists of two hexapods with a collimated red light beam that reflects off the primary and secondary mirrors being aligned. The light beam is viewed by a CCD camera and an optical alignment test known as a Hartmann test is performed several times during the alignment. It is difficult to make a sensible photograph of the alignment station but the CAD graphic below shows the major components.
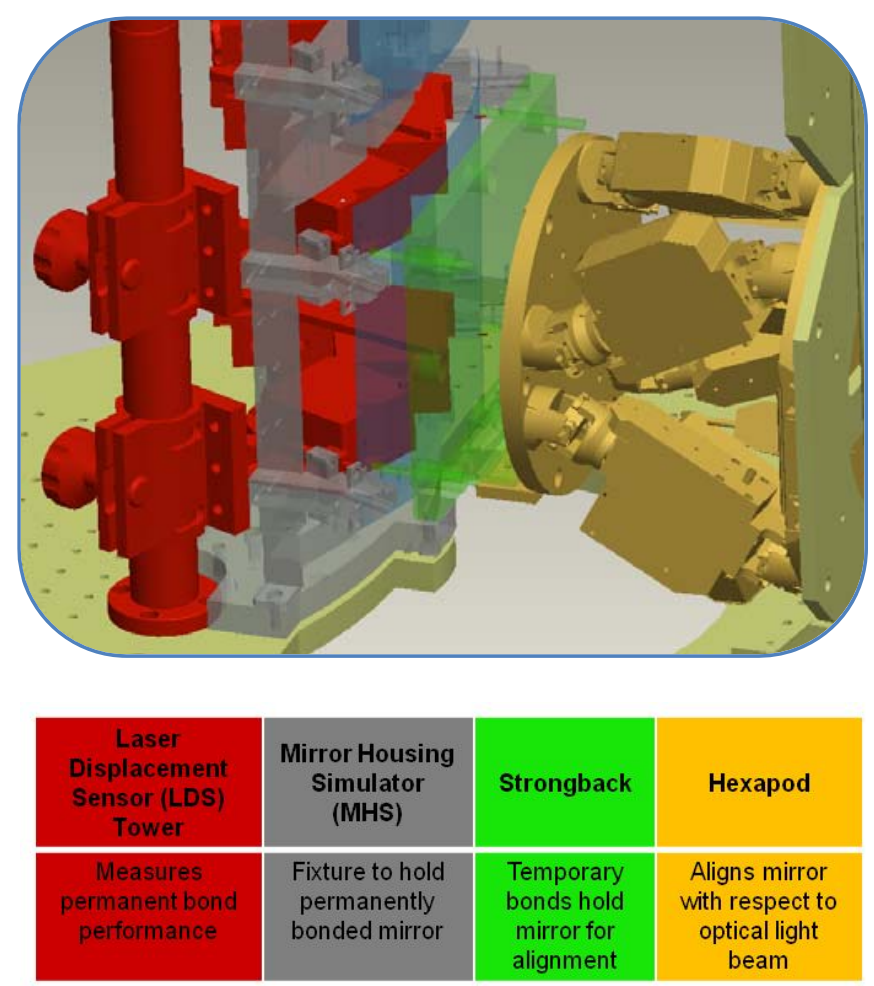

Figure 7 - TRL 4 Optical Alignment Station

The alignment process is summarized as follows:

1. Each hexapod holds an X-ray mirror that has been "temporarily bonded" to several pins protruding from a stiff plate of glass.

2. Hexapods are commanded to move in all 6 degrees of freedom at the sub-micron level to align the primary and secondary mirrors to each other. Hartmann tests establish the alignment.

3. Once aligned, each mirror is bonded to small metal tabs that are secured to the metal housing simulator. Bonding is done while being observed by displacement sensors (laser or capactive sensors) with accuracy of better than $20 \mathrm{~nm}$.

4. As the epoxy shrinks while curing, an algorithm is run to reposition the mirrors back to their initial position.
One major breakthrough in this process was the discovery that the syringe which injects the epoxy can be used as a kind of actuator to reposition the mirrors. The syringe is connected to a nano-actuator and controlled from the displacement sensors. It pushes and pulls the uncured epoxy a few microns back and forth until the mirror returns to its initial position. With this technique, bonds can be made which distort the mirror less than 500 nanometers and this has been found to induce less than 1 arc-second HPD error.

The first successful bonding of a mirror pair in the TRL 4 housing simulator occurred in September 2010. X-ray testing showed that the HPD error of the aligned pair was 12.7 arc-seconds +/- 0.9. Further TRL 4 tests are being performed to repeat and beat this result.

The next step along the way to flight preparedness is to demonstrate TRL 5. The criteria for TRL 5 includes aligning and bonding multiple mirror pairs to better than 10 arc-seconds HPD and then taking the mirror housing through environmental tests including shock, vibration and thermal cycling.

\section{STRUCTURAL, Thermal, Optical ANALYSIS}

Another major challenge to the design of the FMA is the thermal requirements and how best to implement a thermal system to meet them. Based on experience in the TRL 4 development effort, the temperature has a very strong effect on mirror alignment due to thermal expansion effects. For purposes of designing a strawman thermal system, the requirement was proposed of maintaining the temperature of a mirror module at $20 \mathrm{C}+/-0.5 \mathrm{C}$ and between modules at $20 \mathrm{C}+/-1 \mathrm{C}$. The mirror modules will have to be aligned, bonded, tested, and kept at 20C on-orbit.

The "real" thermal requirements are as yet unknown. The specific mirror module design has not been finalized and will probably be firmed up during the TRL 6 technology demonstration effort in the coming years. Once the materials and geometry are better understood, rational thermal requirements will be established on the basis of analysis results.

The IXO project has started a "STOP” analysis (Structural, Thermal, and Optical) as an early effort to bound the thermal requirements. This type of analysis is often performed on an end-to-end optical system to understand how temperature variations and gradients effect the optical quality of the system. A finite element model of the system is developed and a thermal model is overlayed on the model so that displacements can be obtained. These displacements are input into optical modeling software to determine the effect on optical quality such as a loss of focus or other parameters such as cone angle variation, and sag variation.

A mirror module design was modeled in CAD during the 
pre-Phase A effort. The basic outline and geometry of a mirror module is based on the size and shape of the FMA. The module shape is a wedge with curved back and front walls. Its height of about $50 \mathrm{~cm}$ is based on the height of an $\mathrm{X}$-ray mirror pair. The installation and alignment of the mirrors is the tricky part which will be developed in the TRL 5 and 6 technology development effort. Whatever techniques are chosen, it is fairly certain that it will involve 4-8 bond points which are tied to the internal mirror module structure.

A finite element model was made from the mechanical design. Many iterations were performed on the module design to optimize it for mass while retaining sufficiently high bending modes. A software algorithm was developed to generate nodes for each individual mirror. This was a major feat detailed in Reference [3].

A thermal model of IXO was created for the Decadal survey proposal. A more detailed model of the FMA and a mirror module was recently created to predict its gradients and how much heater power is required to maintain it at 20C. Details of this work are beyond the scope of this paper and can be found in Reference [7].

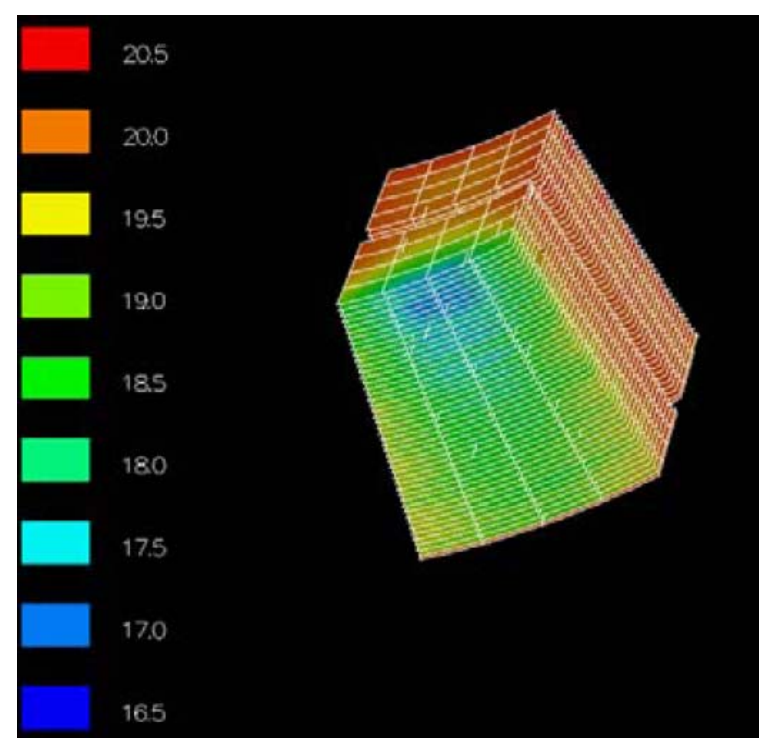

Figure 8 - Typical thermal results for mirrors in a module

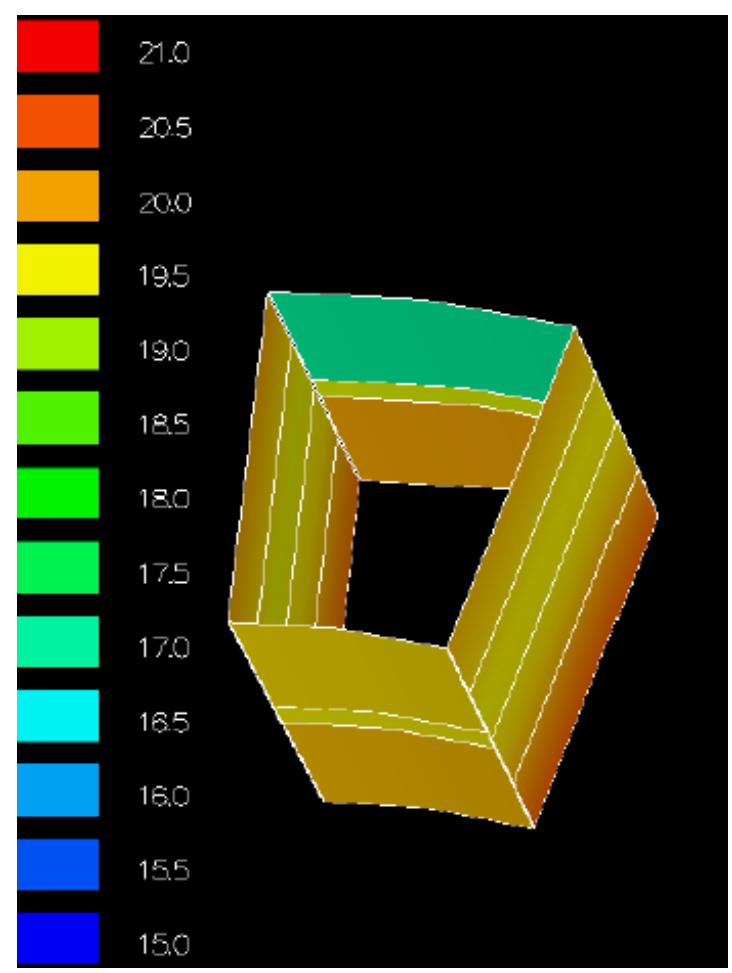

Figure 9 - Typical thermal results for module walls

The main conclusions from this effort are the following:

- $\quad$ Required FMA heater power is less than 900 watts. Heaters on the mirror module exterior walls as well as the FMA primary structure are required to keep the FMA at 20C. Placement is critical.

- The spacecraft adapter which encircles the FMA should be isothermalized by the use of heat pipes and should be heated.

- $\quad 1.6$ meters of the metering structure above the FMA should be heated to $23 \mathrm{C}$ so that the FMA views a warm surface.

- Thermal precollimators will be added on the outward facing side of the mirror modules so that their fields of view are restricted. Precollimators made of G10 or similar non-conducting material will reduce make-up heater power by several thousand watts.

This thermal model with its temperature distribution was mapped onto the structural FEM. Displacements caused by thermal expansion were then calculated and sent to an X-ray optical performance software tool developed at Goddard. The results are still pending. They will consist of a predicted Half Power Diameter error for a mirror module given the predicted thermal gradient within the module that results from the preliminary thermal control system. 


\section{OVERCOMING THERMAL EXPANSION Problems With NeW MATERIALS}

Differential thermal expansion has always been a major challenge for telescope designers. A very common method to overcome differential thermal expansion is to choose optics and structures that have similar coefficients of thermal expansion (CTE). As a telescope made from wellmatched materials warms and cools, the optics and structure grow and shrink together to maintain optical alignment. Optics made from a very low expansion glass such as Zerodur are often mounted in structures made of Invar and CFRP composites which have very low thermal expansion. In the case of IXO, the D263 glass optics have a CTE of 6.3 $\mathrm{ppm} / \mathrm{C}$ at $20 \mathrm{C}$ and so the standard low expansion structural materials are not compatible.

The first task in the materials area was to precisely determine the CTE of D263 glass at 20C. The glass fabricator, Schott, states in its website that the CTE of D263 is $7.2 \mathrm{ppm} / \mathrm{C}$ from $20-300 \mathrm{C}$. This proved to be an average over a large range of temperatures and was not accurate or specific enough around IXO's mirror operating temperature of 20C +/- 1C. For CTE testing, NASA Goddard uses a Michelson Laser Interferometer in an enclosed temperature controlled housing that has been calibrated against a NIST copper standard within .02 ppm/C at 20C. D263 Glass coupons were made and tested in this facility and found to have an average CTE of $6.28 \mathrm{ppm} / \mathrm{C}$ at 20C with a standard deviation of .07C. The tests included several temperature cycles from 10-30C to check for hysteresis which was found to be negligible. Both raw glass and slumped (annealed) glass of different thicknesses were tested and the CTEs were the same within the error of the test apparatus.

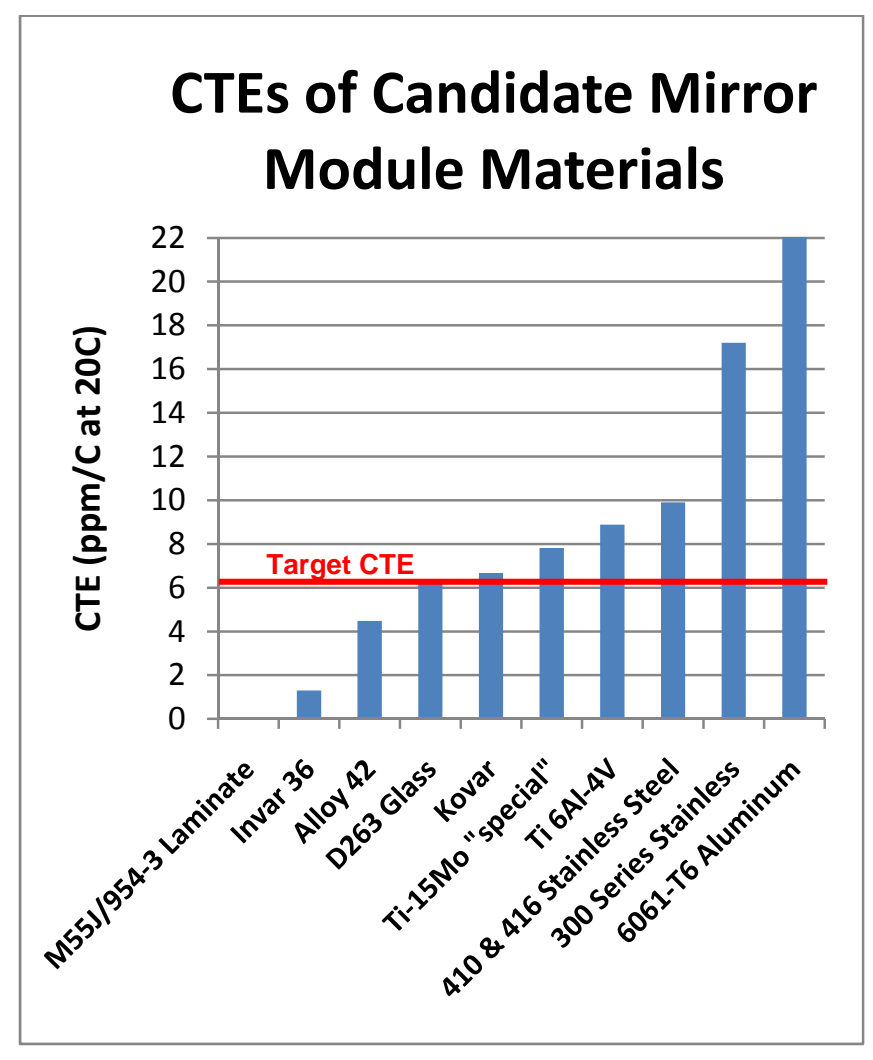

Figure 10- CTE chart

A precise CTE measurement was also made on Kovar coupons. Kovar is a tradename for UNS K94610, and another tradename is Nilo Alloy K. It is an alloy of Iron, Nickel, and Cobalt that is commonly used as a glass-tometal seal for transistors and electronics. The strength and density of Kovar are similar to Invar or mild steel which is not especially favorable for aerospace structures. The CTE of annealed Kovar was found to be $6.67 \mathrm{ppm} / \mathrm{C}$ at 20C with a standard deviation of .08C. Later tests of "as-is" off the shelf Kovar came in at $6.54 \mathrm{ppm} / \mathrm{C}$ at $20 \mathrm{C}$ which was an even better match to D263 glass.

A study was made to understand the sensitivity that an IXO mirror module has to differential thermal expansion. A finite element model of a mirror module was combined with an in-house X-ray optical analysis code. Node displacements and rotations caused by a bulk temperature change or a temperature gradient in the mirror module were output by NASTRAN and translated into optical errors in terms of arc-seconds of "Half Power Diameter" (HPD) error. This kind of analysis is essentially a STOP analysis with simplified thermal conditions. 


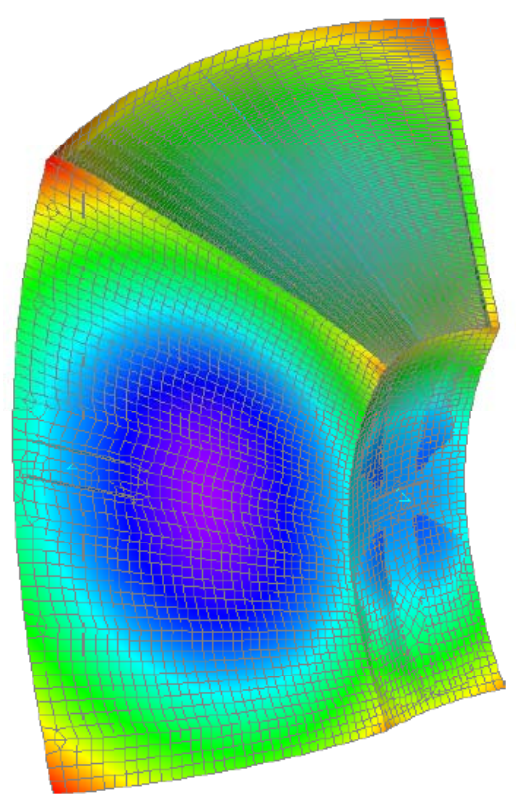

Figure 11 - Distortions on a mirror module due to thermal expansion from a temperature gradient mapped onto the module FEM.

The following figure shows the effect that a bulk temperature change of a mirror module has on optical error versus different candidate module structural materials. The end-to-end requirement for IXO is 5 arc-seconds HPD and the error budget for thermal effects is about 1 arc-second HPD.

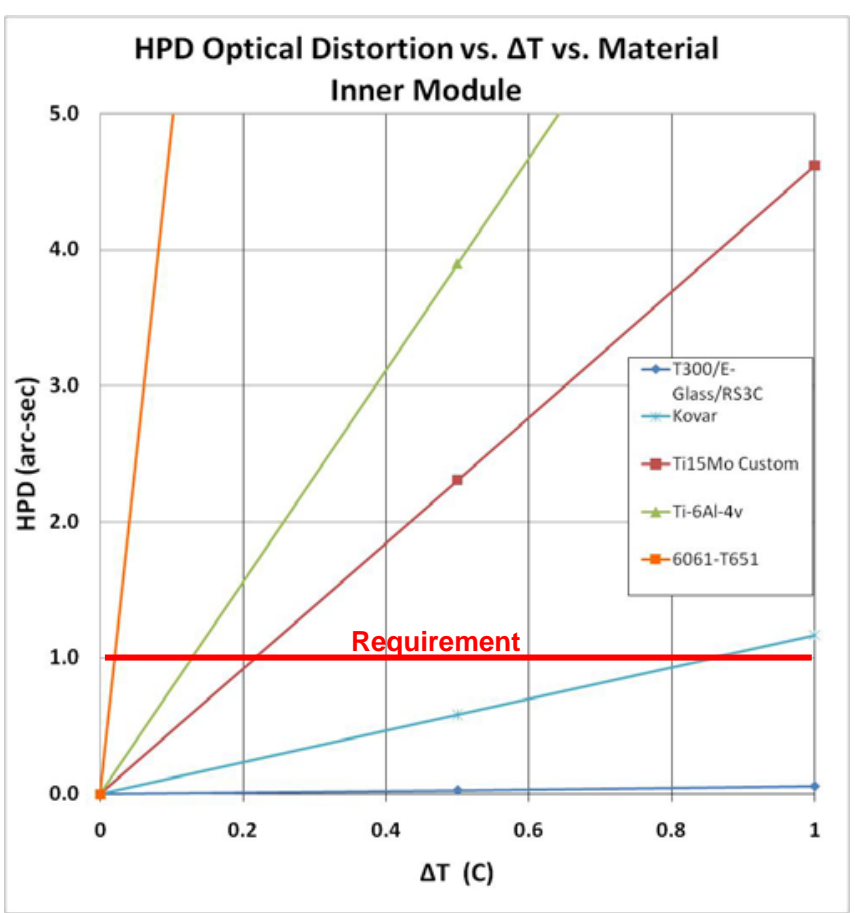

Figure 12 - Distortion vs. temperature vs. Material choice

As the chart shows, depending on the mirror module housing material, the allowable operating temperature range is extremely limited if the housing is made of aluminum, $0.2 \mathrm{C}$ if made from Ti-15Mo alloy, or $0.8 \mathrm{C}$ if made from Kovar. The IXO project decided to use Kovar where possible in the mirror module technology development efforts. It is the most compatible metal alloy to use with the $\mathrm{X}$-ray mirrors.

If a mirror module material could be found with matching thermal expansion to D263 glass then the problems of temperature control might be avoided, and the easier it will be to implement the FMA thermal control system.

IXO has started a project to obtain samples of a modified form of Kovar which has its Iron and Nickel ratios altered slightly to lower the CTE into the $6.3 \mathrm{ppm} / \mathrm{C}$ range. This work is ongoing.

Another ideal material for the mirror module might be a CFRP (Carbon Fiber Reinforced Plastic) composite laminate with a CTE that matches D263 glass. CFRP materials have a much lower density than Kovar and higher strength and stiffness. Typical CFRP laminates have CTEs in the range of $0-2 \mathrm{ppm} / \mathrm{C}$. No common laminates were known to have a CTE near $6.3 \mathrm{ppm} / \mathrm{C}$, so a development effort was started at GSFC to see if one could be developed.

Using standard laminate theory, we determined that adding E-glass (a form of fiber glass) layers to T300 carbon fiber would raise the CTE to the desired level. RS-3C resin was selected as being a good match for both fibers. The T300/RS-3C prepreg (pre-impregnated fiber) and Eglass/RS-3C are fairly common prepregs in the composite industry and not difficult to obtain. Another important requirement is that the laminate be quasi-isotropic and have the same CTE in both in-plane directions. The out-of-plane direction (through the thickness) cannot be made to match the CTE of the in-plane laminate due to the nature of the fibers. This should not be an obstacle because the module can be designed to allow the CRFP panels to expand away from the glass interfaces when thermal gradients are present.

The lamina design to achieve $6.3 \mathrm{ppm} / \mathrm{C}$ was determined to be eight plies of E-glass/RS3C and eight plies of T300/RS3C prepregs at $\left[0^{1} / 0^{2} / 45^{1} / 45^{2} / 90^{1} / 90^{2} /-45^{1} /-45^{2}\right]_{\text {S }}$, where subscripts 1 and 2 denote E-glass and T300 layers, respectively. Assuming each layer to be .005" thick, the total thickness of the laminate was predicted to be .080 " ( $\sim \mathrm{mm})$. If thicker laminates are desired, they can be made in increments of .080 " by repeating this lamina schedule. It is also possible to create another thinner quasi-isotropic layup utilizing a $[0 / \pm 60]$ layup, but this was not investigated in this project.

The overall CTE of laminates is a balance of the thermal expansion of the fiber and the resin. Carbon fiber has a low or even negative CTE while resin CTEs are much higher. Prepregs can be made "resin rich" or "resin poor" and a specific amount of resin can be bled out through the curing process to achieve a target CTE. The key variable to this 
laminate is to determine the fiber volume (FV) to achieve $6.28 \mathrm{ppm} / \mathrm{C}$. In addition to fiber volume, many properties have a direct influence on the CTE such as fiber tow, tack, (arrangement and packing of the fibers in the prepreg), void fraction, fiber diameter, modulus, strength, and density. The budget for this effort was not sufficient to pay for material property tests of the fiber, resin, and prepregs prior to fabricating the laminate coupons which would allow an accurate determination of the fiber volume required prior to fabricating a laminate. Several laminates were made to envelope or bracket the fiber volume required given this particular supply of prepreg material.

Fiber volume can be controlled initially during the manufacture of the prepreg material by allowing more or less resin to mix with the fibers. During laminate manufacturing, $\mathrm{FV}$ is controlled within $1-2 \%$ using special bleeder cloths which absorb the resin a given amount during the curing process. Controlling resin bleedout better than this level is not possible without extensive process controls not available to IXO.

Several $12 \times 12$ " coupons were fabricated to different fiber volumes and coupons were cut from these laminates and tested in Goddard's CTE apparatus. Matrix digestion tests were done to determine the precise fiber volume of each coupon. The lowest FV achieved with these prepregs was about $52 \%$ and the best CTE achieved at this FV was 6.0 $\mathrm{ppm} / \mathrm{C}$ at 20C.

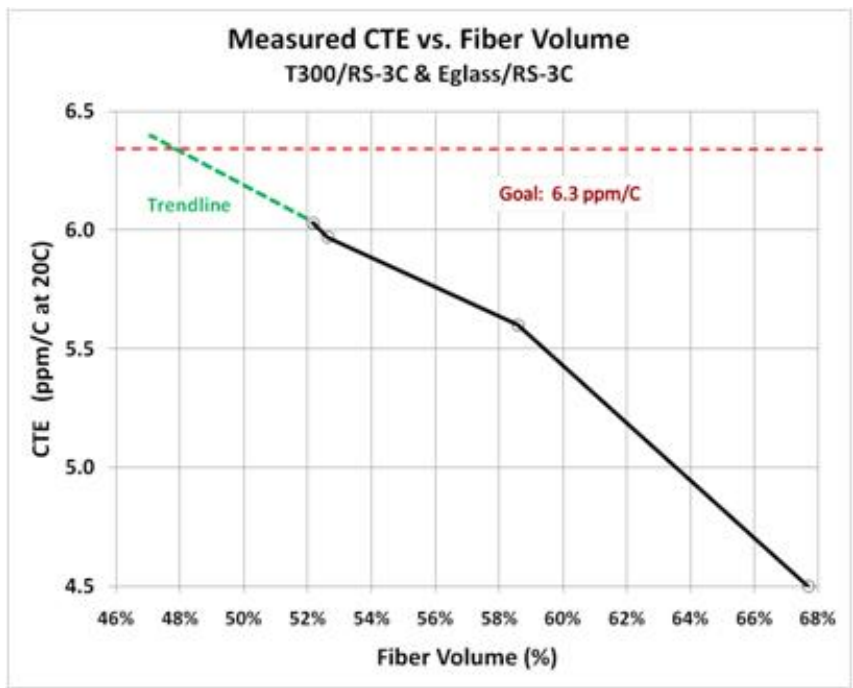

Figure 13 - CTE vs. Fiber Volume Results

Looking at the trendline in the figure above, it can be seen that a fiber volume near $48 \%$ will provide the desired CTE of $6.3 \mathrm{ppm} / \mathrm{C}$. The prepregs on hand were not quite resin-rich enough to reach $48 \% \mathrm{FV}$. After achieving the highest CTE possible with these coupons, they were tested for their mechanical properties which can be seen below.

\begin{tabular}{|c|c|}
\hline Property & Value \\
\hline CTE at 20C (ppm/C) & 6.0 \\
\hline Ultimate Tensile Strength & $\begin{array}{l}67 \text { ksi, std dev .2.5 } \\
\text { (465 Mpa) }\end{array}$ \\
\hline $\begin{array}{l}\text { Ultimate Compressive } \\
\text { Strength }\end{array}$ & $\begin{array}{l}66 \text { ksi, std dev } 2.8 \\
\text { (455 Mpa) }\end{array}$ \\
\hline Density & $\begin{array}{l}.0616 \text { lb/in3 } \\
\text { (1705 kg/m3) }\end{array}$ \\
\hline Young’s Modulus (E) & $\begin{array}{l}\text { 4.7 MSI, std dev } 0.04 \\
\text { (32.4 Gpa) }\end{array}$ \\
\hline Poisson’s Ratio & 0.31 , std dev 0.01 \\
\hline $\begin{array}{l}\text { Fiber Volume for these } \\
\text { coupons }\end{array}$ & $52 \%$, std dev 0.97 \\
\hline
\end{tabular}

It is useful to compare the properties of this laminate with the standard M55J zero-CTE laminate as well as Ti6Al-4V Titanium, and Kovar. These are the most likely candidates for mirror module structure or piece parts.

\begin{tabular}{|c|c|c|c|c|}
\hline Property & $\begin{array}{l}\text { T300/ } \\
\text { Eglass }\end{array}$ & $\begin{array}{l}\text { M55J/ } \\
954\end{array}$ & $\begin{array}{l}\text { Ti- } \\
6 \text { Al- } \\
4 \mathrm{~V}\end{array}$ & Kovar \\
\hline CTE at 20C & 6.3 & -0.2 & 8.9 & 6.7 \\
\hline $\begin{array}{l}\text { Density } \\
\text { (kg/m3) }\end{array}$ & 1705 & 1688 & 4430 & 8359 \\
\hline $\begin{array}{l}\text { Young's } \\
\text { Modulus } \\
\text { (GPa) }\end{array}$ & 32.4 & 138 & 114 & 138 \\
\hline $\begin{array}{l}\text { Tensile } \\
\text { Yield } \\
\text { Strength } \\
(\mathrm{MPa})\end{array}$ & 465 & 345 & 880 & 345 \\
\hline $\begin{array}{l}\text { Stiffness/ } \\
\text { Density } \\
\text { ratio }\end{array}$ & .019 & .062 & .026 & .017 \\
\hline $\begin{array}{l}\text { Strength/ } \\
\text { Density } \\
\text { ratio }\end{array}$ & .273 & .204 & .199 & .041 \\
\hline
\end{tabular}


A bolded number indicates the most favorable property among the candidates. This laminate's best attribute, besides matching the CTE of D263 glass, is that its strength to density ratio ("specific strength") is much higher than the others. Its least favorable attribute is the Young's Modulus of $32.4 \mathrm{GPa}$ which is quite low. This means that more material (more moment of inertia) will be required in a load bearing structure to maintain a high enough natural frequency than if more typical aerospace materials were used. On the plus side, its specific stiffness is slightly better than Kovar which is the leading candidate metal for the X-ray mirror module structure.

This laminate may be the only composite material in existence that matches the CTE of D263 glass. This helps simplify the thermal system by allowing a larger temperature operating range on the FMA. Better coupons can be made when prepregs with more resin can be obtained. Achieving a precise CTE can be done given that extensive process controls are put in place at the prepreg manufacturer and during the laminate consolidation phase to control all the variables.

\section{Preliminary Coupled loads Analysis}

A preliminary coupled loads analysis (CLA) was performed of the observatory on an Atlas 551 expendable launch vehicle. This type of analysis consists of creating a finite element model of the observatory and combining it with a FEM of the launch vehicle and subjecting the models to launch loads. It is somewhat unusual to perform a CLA at this early stage of a project, but it was deemed necessary to help inform the technology development efforts of the flight mirror assembly and the focal plane instruments. The launch vehicle side of the coupled loads analysis was performed by the analysis group at the Kennedy Space Center and results sent to Goddard for post-processing.

Getting the CLA results this early is very helpful and will result in mass savings since most instruments are developed to loads obtained from generic Mass Acceleration Curves or sine base drives which are sometimes overly conservative.

The CLA results confirmed that the $1^{\text {st }}$ bending mode of the observatory was $>8 \mathrm{~Hz}$ and that all significant axial modes were $>27 \mathrm{~Hz}$. Also several areas in the structure were shown to be insufficiently stiff or weak. Fortunately these areas were minor and the addition of ribs or thickening a facesheet resolved these problems. The results were very encouraging and showed that the overall design concept of the observatory is reasonable from the stress and stiffness point of view. Also it validated the structural mass estimate.

Prior to the receiving the CLA results, one area of uncertainty was whether the low FMA torsion mode would be excited or not. Because its construction is similar to a wagon wheel or spider web, a low torsion mode exists in the design around $18 \mathrm{~Hz}$. It would be easy to increase this mode but would require diagonal braces which would obscure some of the clear aperture of the mirror assembly which is obviously undesirable, and it adds mass.

Fortunately the CLA results showed that the torsion mode was not appreciably excited. The Atlas launch vehicle and flight trajectory does not induce modes that can couple into the FMA torsion mode.

Another area of interest is the loads at the bond sites of the mirror. Knowing the loads at these points is necessary to establish the surface area required at each bond site, the number of bond sites, and to help to select the most favorable epoxy.

CLA results for the loads at the mirror module flexures were recovered and input into a more accurate mirror module FEM. The FMA mirror module quasi-static net CG load levels are 6.14 G's axial and 1.69 G's lateral. These are much lower than the 18 G's previously assumed in initial calculations for mirror stress and bond sizing. The current arrangement of bond sites (three on each side and one at top and bottom of each mirror) is adequate and provides a positive margin of safety. It may be possible in future to reduce the number of bond sites when specific strength allowables are developed for the tab-to-glass bond. Also, the low results are helpful for future mirror module structural design and STOP analyses.

IXO's suite of instruments are in the early stages of development. The CLA results for the instrument module were determined to be 7.35 G's axial, 1.25 G's lateral. These loads are so low that they may be exceeded by transportation loads. Working to these loads in pre-Phase A instead of a 14 or $18 \mathrm{G}$ quasi-static estimate will definitely result in mass savings.

\section{LAUNCHER COMPARISON}

As part of the collaboration between NASA, ESA, and JAXA, a study was performed to understand the differences between using an American EELV (Evolved Expendable Launch Vehicle) and the Ariane 5. On the US side, there are currently two candidate EELVs including the Delta IV and the Atlas V. The Delta IV medium does not have sufficient payload capability for IXO and the Delta IV Heavy is much too capable and costly for the project. That leaves the Atlas $\mathrm{V}$ as the most likely candidate, and the Atlas 551, the largest version, is the current baseline launch vehicle for purposes of the IXO study. On the ESA side, the Ariane 5 is the only choice.

The table below shows a comparison of the environments between the Atlas $\mathrm{V}$ and the Ariane 5 launchers as specified by their respective planner's guides [9], [10]. 


\begin{tabular}{|c|c|c|}
\hline Environment & Atlas - V & Ariane 5 \\
\hline Lateral DLL (G) & 2 & 2 \\
\hline Axial DLL (G) & 6 & 6 \\
\hline $\begin{array}{l}\text { Sine Vibration } \\
\text { Lateral }(G)\end{array}$ & $\begin{array}{l}0.8 \mathrm{G} \text { from } 5-25 \\
\mathrm{~Hz} ; \\
0.7 \mathrm{G} \text { from 25- } \\
100 \mathrm{~Hz}\end{array}$ & $\begin{array}{l}0.8 \mathrm{G} \text { from } 5-25 \\
\mathrm{~Hz} \\
0.6 \mathrm{G} \text { from 25- } \\
100 \mathrm{~Hz}\end{array}$ \\
\hline $\begin{array}{l}1^{\text {st }} \text { Bending mode } \\
(\mathrm{Hz})\end{array}$ & 8 & 8 \\
\hline $1^{\text {st }}$ Axial mode & 15 & 27 \\
\hline $\begin{array}{l}\text { Acoustics } \\
\text { OASPL (dB) }\end{array}$ & 140.3 & 139.5 \\
\hline Shock & Mission specific & Mission specific \\
\hline
\end{tabular}

There is very little difference between launchers in terms of loads, with the exception of the $1^{\text {st }}$ axial mode. The Ariane 5 requires a much higher axial mode $(27 \mathrm{~Hz})$ compared to Atlas V $(15 \mathrm{~Hz})$. Because of the large diameter and mass of the FMA, it is susceptible to having a low drum-head axial mode if careful design is not applied. This drum head mode is overcome by using deep but thin beams for the primary structure of the mirror and the current design has a drum head mode above $50 \mathrm{~Hz}$.

The main difference between launch vehicles is the availability of large scale payload adapters. IXO is designed with its heavy FMA down at the bottom of the spacecraft next to the payload adapter to help lower the observatory center of gravity which in turn helps to keep the bending modes above the required $8 \mathrm{~Hz}$. IXO's CG is at about $5 \mathrm{~m}$ above the separation plane which is much higher than most satellites. Another desirable feature for IXO's payload adapter would be to have a large base of support for the $8 \mathrm{~m}$ tall observatory. The larger base of support spreads the observatory's loads over a larger area within the adapter so that it may be able to handle IXO's high CG. Since the diameter of the optics module is about $3.2 \mathrm{~m}$, it is highly desirable to have a payload adapter about this diameter.

The largest standard payload adapter offered by the Atlas $\mathrm{V}$ has a $1.66 \mathrm{~m}$ diameter, but with a payload of IXO's mass the CG height limitation is $\sim 2.5 \mathrm{~m}$ which is not sufficient. Even if it was sufficient, IXO would require a conical, or frustum shaped adapter ring to adapt from $1.66 \mathrm{~m}$ to $3.2 \mathrm{~m}$ diameter and mass estimates for this large adapter run in the 100-200 kg range.
Fortunately the Atlas V mission planner's guide offers the 3302 Truss Adapter. This is a $3.3 \mathrm{~m}$ diameter "elephant stand" type of adapter attaches to the Centaur outer ring instead of the Centaur's smaller upper platform where the standard adapters interface. The 3302 Truss Adapter also has the CG height capability for IXO so it is the obvious choice for IXO. The main disadvantage of the 3302 is that although it was qualified as part of the EELV program, it has not yet flown. In addition there is a $194 \mathrm{~kg}$ launch mass penalty when using it.

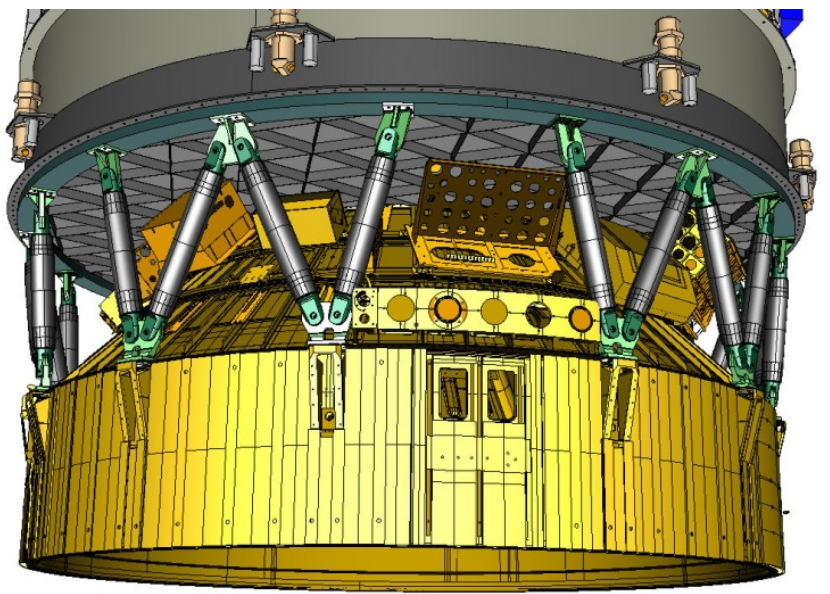

Figure 14 - View of the 3302 Truss Adapter (credit: United Launch Alliance)

On the Ariane side, there are two choices of payload adapters. Ariane 5 offers the 2624 payload adapter which has a basic diameter of $2.624 \mathrm{~m}$ and a modified "SDM" adapter. The 2624 adapter has a CG height capability of about $5.2 \mathrm{~m}$. A frustum adapter would be required to adapt from $2.624 \mathrm{~m}$ to $3.2 \mathrm{~m}$ of the FMA and this adapter is estimated to weigh an additional $200 \mathrm{~kg}$.

ESA's Separation and Distancing Module (SDM) was developed for the Jules Verne Automated Transfer Vehicle to the space station. It successfully flew in March 2008. The SDM has a diameter of $3.9 \mathrm{~m}$ and an adequate CG height requirement for IXO. The SDM is about $2 \mathrm{~m}$ tall which is much taller than necessary for IXO and so it is likely that the design could be modified by reducing its height to about $50 \mathrm{~cm}$. Its diameter is too large for IXO to use directly and so a frustum adapter would be required to adapt from $3.9 \mathrm{~m}$ to the $3.2 \mathrm{~m}$ FMA. This adapter is expected to weigh about $200 \mathrm{~kg}$. 


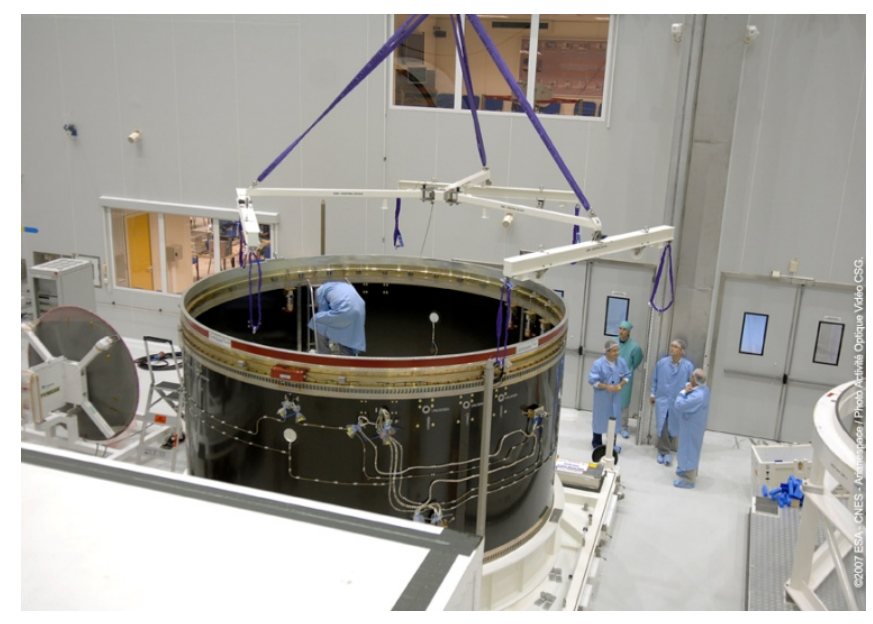

Figure 15 - ESA's SDM being prepared for use on ATV-1.

To summarize the results of the launch vehicle study, it is definitely possible to fly IXO on either an American EELV such as the Atlas V or the European Ariane 5. In both cases a mission unique payload adapter will be required but they will be based on existing qualified designs.

\section{REFERENCES}

[1] J. Bookbinder, R. Smith, A. Hornschemeier, et al, "The Constellation-X Observatory” Proc. of SPIE Vol. 7011, 701102, 2008.

[2] R. McClelland and D. Robinson, "Design Concept for the International X-Ray Observatory Flight Mirror Assembly,” 2009 IEEE Aerospace Proceedings, March 613, 2009.

[3] McClelland, R., Robinson, D., Design and Analysis of the International X-Ray Observatory Mirror Modules, Proc of SPIE Vol 7732. (2010).

[4] K. Wallace, M. Bavdaz, P. Gordon, "Silicon pore optics development” Proc. Of SPIE, 7437 (2009).

[5] J. Koglin, F. Christensen, W. Craig, et al, "NuSTAR hard X-ray optics” Proc. Of SPIE 5488, 856 (2004).

[6] Evans, T., Chan, K., Saha, T., Bonding Thin Mirror Segments without Distortion for the International X-Ray Observatory, Paper 1336, 2011 IEEE Aerospace Proceedings, March 5-12, 2011.
[7] Choi, M., "Thermal Optimization of IXO SXT FMA to Meet $20^{\circ} \mathrm{C}$ and Stringent Temperature Gradient Requirements and Heater Power Budget” AIAA-20106054, 40th International Conference on Environmental Systems, Barcelona, Spain, July 11-15, 2010.

[8] "New Worlds, New Horizons in Astronomy and Astrophysics," Committee for a Decadal Survey of Astronomy and Astrophysics, National Research Council, The National Academies Press, Washington, DC, 2010.

[9] Atlas Launch System Mission Planner’s Guide, Version 10A, January 2007.

[10] Ariane 5 User’s manual issue 5, Revision 0, July 2008.

\section{BIOGRAPHY}

David Robinson is the Lead Mechanical Systems engineer

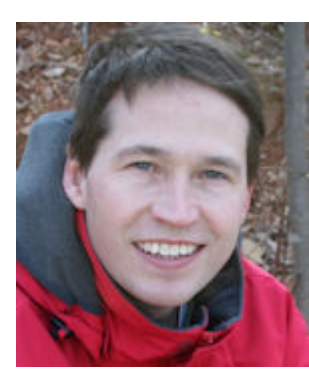
of the IXO Project at the NASA Goddard Space Flight Center. He started his career with NASA Glenn Research Center in 1990 working on the International Space Station and several microgravity fluids space experiments for the space shuttle and the Russian Mir space station. While at Goddard, he worked on JWST, Swift, the recently launched Solar Dynamics Observatory and many proposals along the way. He received a B.S in Aerospace Engineering from the University of Virginia, a M.S. in Mechanical engineering at Cleveland State University, and a M.S. in Space Studies at the International Space University in Strasbourg, France. 